\title{
Variable Input Coupler Design for Storage Ring Cavities
}

\author{
Y. W. Kang, and R. L. Kustom \\ RF Group \\ Accelerator Systems Division \\ Advanced Photon Source
}

August 18, 1995
RFAFIVED

JAN 251995

USTI

Magnetic loop type input couplers are used for coupling if power from waveguides to the storage ring cavities: In a high if power and high beam current accelerating cavity, the change in beam loading results in high reflected power due to input if mismatch. The coupler can be matched for a specific loading condition, but cannot be matched in other conditions. The input mismatch results in poor rf power efficiency and overheating of the ceramic window in the coupler. Therefore, coupling through the coupling loop must be adjustable for maximum operating power efficiency and coupler reliability.

The adjustment of coupling can be made by changing the magnetic flux linkage through the loop area. This can be done either mechanically by moving the coupling loop position or electronically by using impedance matching to change the properties of low loss material such as ferrite. In the existing coupler design, to change the coupling the coupler loop is turned physically for matching. The cavity vacuum must be broken and pumped down again; this can cause long system down time.

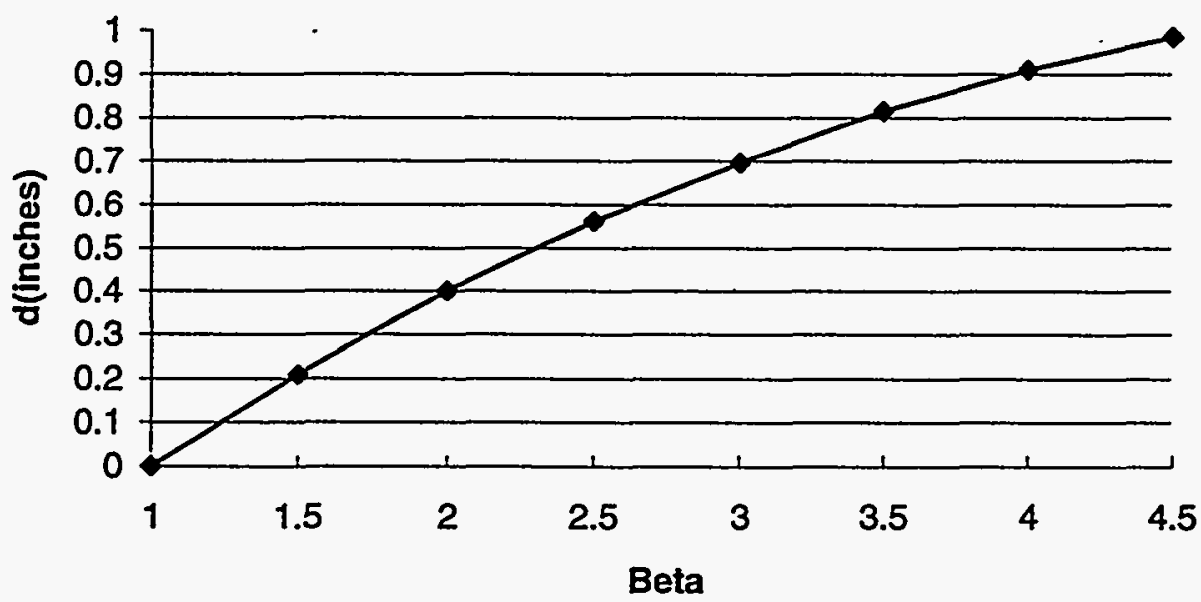

Figure 1 -- Loop displacement, d, vs. cavity $\beta$ 
Cavity $\beta$ is defined as

$$
\beta=\frac{Q_{o}}{Q_{L}}-1
$$

where $Q_{0}$ and $Q_{L}$ are the unloaded $Q$ and loaded $Q$ of the cavity, respectively. The single-cell cavities in the storage ring of the APS have $Q_{0} \approx 49,000$ and $Q_{L} \approx 10,000$ under the anticipated maximum beam loading condition [1]. Measurement of a prototype cavity shows that, in a $352 \mathrm{MHz}$ single-cell cavity, the coupler can be impedance matched for $\beta=1$ to 4.0 by moving the loop position about 0.9 " [2]. The beam loading was simulated by damping the fundamental accelerating mode resonance. Figure 1 shows the coupler loop position vs. $\beta$. For higher $\beta$, the loop must move into the cavity for more flux linkage. A feedback control loop can be installed in the low-level if system for independent automatic control of the input cavity coupling. The feedback system monitors the impedance matching condition and minimizes the input reflection by adjusting the position of the coupler loop.

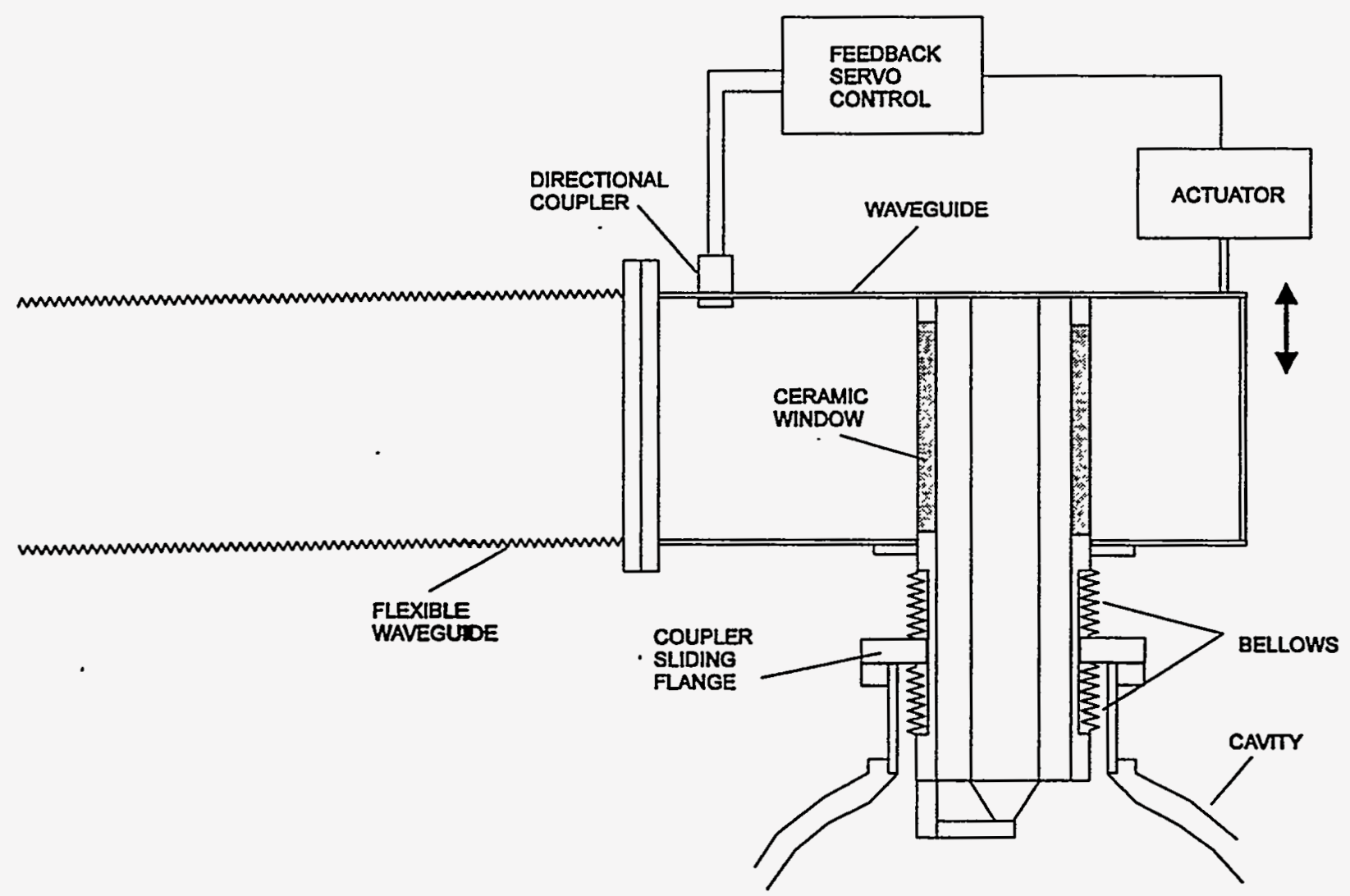

Figure 2 -- A variable input coupler 
A continuously variable coupler design that can be used under vacuum and high power operating condition is shown in Figure 2. A flexible waveguide section is needed in this design to realize the adjustable coupler structure. The flexible waveguide works as the flexible arm for moving the coupler. If the distance from the ceramic window to the farther end flange of the flexible waveguide is greater than 4 or 5 feet, moving the loop coupler by 1" would not be stressful to the flexible waveguide. The servo actuator can be controlled with a feedback loop for optimum matching for a varying beam loading condition. The bellows are used outside the outer conductor of the coaxial transmission line section of the coupler. The bellows ensure that the coaxial section has no vacuum-related mechanical changes during variable matching.

The details of the bellows and the sliding flange are shown in Figure 3. The vacuum flange of the coupler is welded or brazed to two separate bellows but not to the coupler body: one is inside the cavity port and the other one is outside the cavity port. The vacuum flange is not attached to the outer cylinder of the coaxial coupler structure and may move freely. The bellows inside the cavity port functions as the vacuum barrier. The space between the bellows and the outer cylinder of the coaxial section can be filled with air; the bellows may be cooled with forced air in that space.

The rf power dissipation in the annular gap between the cavity port cylinder and the coupler outer cylinder is small. The accelerating $\mathrm{TM}_{01}$ mode has almost no coupling to this area since the coupler is located in the cavity midplane. Thus, $\mathrm{rf}$ heat dissipation on the surface of the bellows will be relatively negligible. However, smaller surface area of the bellows is better for less if heating. The inner and the outer coaxial conductors can be water cooled.

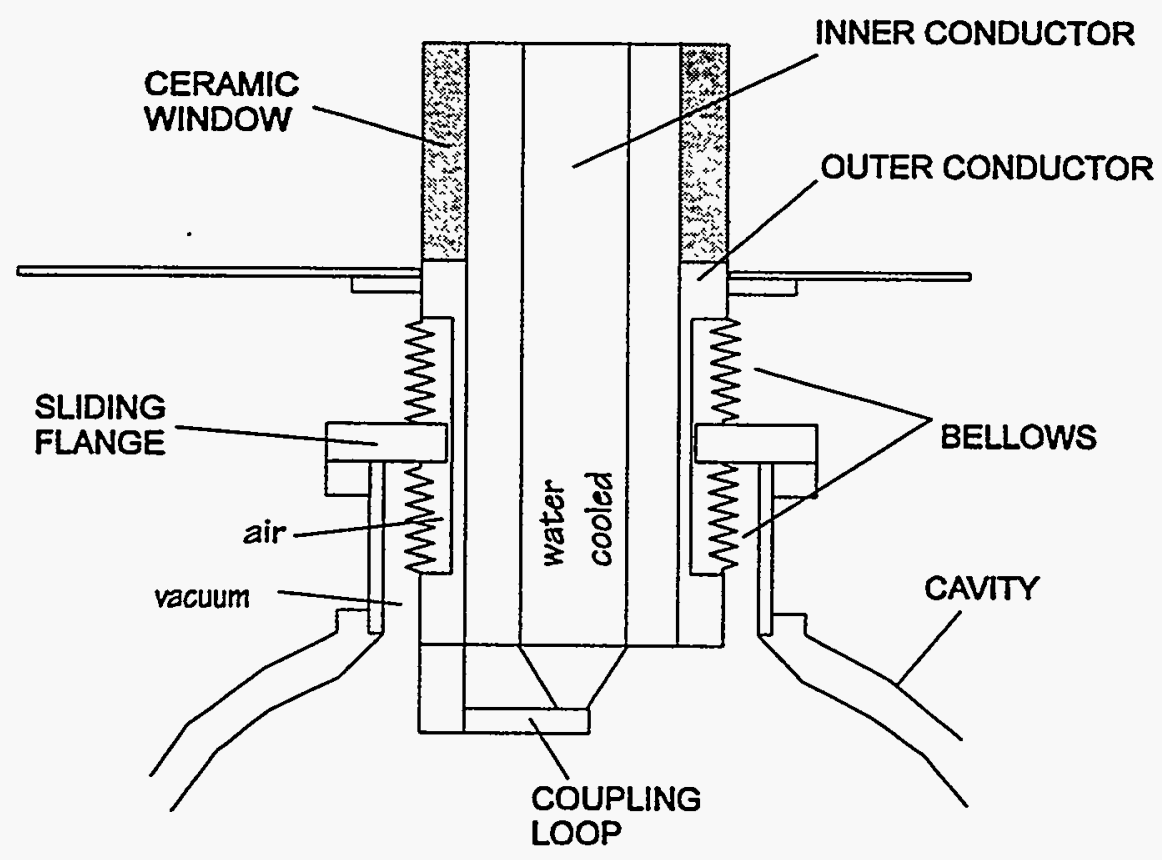

Figure 3 -- Close-up of the area of bellows, flanges, and coupling loop 


\section{REFERENCES}

[1] 1.3.1.4 Storage Ring RF Systems, APS RF Design Handbook.

[2] J. Cho and Y. Kang, "Measurement of Input Coupler Matching of a Loaded Storage Ring Single-Cell Cavity," Light Source Note LS-247, August 14, 1995. 